\title{
UMA METODOLOGIA DE ENSINO DE GEOMETRIA A PARTIR DE UM JOGO DE DOMINÓ: UMA ATIVIDADE LÚDICA DE MATEMÁTICA NA EDUCAÇÃO BÁSICA
}

\section{Marcus Vinicius Sandoval Paixão \\ Frederico César Ribeiro Marques \\ Maria das Graças Carmo}

Instituto Federal do Espírito Santo - Campus Santa Teresa

Santa Teresa, Espírito Santo, Brasil

E-mail:mvspaixao@bol.com.br, frederico.marques@ifes.edu.br,mdcarmoc1@yahoo.com.br

\section{Resumo:}

O objetivo deste trabalho foi o de planejar e aplicar um jogo de dominó matemático para o ensino de geometria, englobando a temática de geometria plana e especial, como estratégia de complementação dos conteúdos programáticos de ensino de matemática do ensino médio. A partir da atividade lúdica, busca-se promover reflexões a cerca das relações matemáticas estabelecidas no cálculo da área de figuras planas e de volume de sólidos de resolução, contribuindo para melhoria do processo de ensino-aprendizagem de geometria. A pesquisa qualitativa sobre uma atividade pedagógica lúdica de ensino de geometria foi realizada no contexto do ensino técnico de nível médio. Os sujeitos desta pesquisa foram estudantes de uma turma do terceiro ano de ensino técnico integrado ao ensino médio de uma escola da Rede Federal de Educação Tecnológica do Espírito Santo, localizada na cidade de Santa Teresa, Estado do Espírito Santo, Brasil. Os dados foram produzidos a partir de observações, questionários e relatórios da aplicação do jogo de dominó matemático. Os resultados indicaram que o jogo de dominó se constituiu em uma atividade lúdica oportunizando a melhoria do processo de ensino-aprendizagem de conteúdos programáticos de geometria no ensino de matemática.

\section{Palavras-Chave:}

ensino de matemática, atividade lúdica, jogo de dominó matemático, geometria, educação básica.

\section{A METHODOLOGY OF GEOMETRY EDUCATION FROM A DOMINO GAME: A LADICAL ACTIVITY OF MATHEMATICS IN BASIC EDUCATION}

\section{Abstract:}

The objective of this work was to plan and apply a mathematical domino game for the teaching of geometry, encompassing the theme of plane and special geometry, as a strategy to complement the programmatic contents of high school mathematics teaching. From the play activity, it is sought to promote reflections about the mathematical relationships established in the calculation of the area of plane figures and volume of resolution solid, contributing to the improvement of the teaching-learning process of geometry. The qualitative research on a pedagogical activity of geometric teaching was carried out in the context of technical education of medium level. The subjects of this research were students of a third year of technical education integrated to the high 
school of a school of the Federal Network of Technological Education of Espírito Santo State, located in Santa Teresa city, Espírito Santo State, Brazil. The data were produced from observations, questionnaires and application reports of the mathematical domino game. The results indicated that the domino game constituted a playful activity, facilitating the improvement of the teachinglearning process of programmatic contents of geometry in the teaching of mathematics.

\section{Keywords:}

teaching mathematics, play activity, mathematical domino game, geometry, basic education.

\section{Introdução}

De acordo com as Diretrizes Curriculares Nacionais da Educação Básica (BRASIL, 2013), a matemática se constituiu em uma das ciências consideradas como base educacional para o processo de ensino-aprendizado na formação do individuo desde o primeiro ano da educação básica. Trata-se de uma linguagem para compreender o mundo, fundamental no processo de construção de cidadania.

No âmbito da escola, com a publicação das Orientações Curriculares Nacionais para o Ensino Médio (OCNEM) da área das Ciências da Natureza, Matemática e suas Tecnologias (BRASIL, 2006), metodologias de ensino foram ampliados e ganharam maior importância no cenário da educação básica brasileira. Um dos desafios no ensino da matemática é a [...] abordagem de conteúdos para resolução de problemas, tratando-se de uma metodologia pela qual o estudante tem oportunidade de aplicar conhecimentos matemáticos adquiridos anteriormente. A proposta de metodologias alternativas para ensinar matemática, também é abraçada pelas OCNEM, incluindo de jogos pedagógicos e outras atividades lúdicas. No caso deste trabalho, trouxemos a proposta do jogo de dominó, pois é uma atividade conhecida pelos estudantes e por possuir regras simples, o que pode agregar potencialidades pedagógica no processo de ensino-aprendizagem do conteúdos de matemática. Esta característica do jogo pedagógico afina-se com a possibilidade de promover sociabilização, além de agregar os conteúdos programáticos.

O jogo pedagógico como instrumento facilitador da aprendizagem de adolescentes tem sido estudado e analisado por vários estudiosos do processo de ensino-aprendizagem. Alguns estudos demonstram que além de propiciar a construção de um espaço no imaginário das crianças e jovens, o jogo, [...] por ser uma atividade dinâmica capaz de transformar-se com o contexto, além de agir como um recurso pedagógico é sugerida como facilitadora do processo de ensino/aprendizagem e do desenvolvimento físico, cognitivo e intelectual infantil (DIVA MARANHÃO, 2004).

O ensino-aprendizagem no decorrer dos anos vem sofrendo mudanças na metodologia de ensino buscando formas que facilitem o trabalho do professor no processo de aprendizagem. As mudanças referentes ao $\mathrm{s}$ 
recursos didáticos, principalmente os pedagógicos, incluem os jogos que, quando usados adequadamente tornam a aprendizagem menos mecânica e mais significativa e prazerosa para o estudante, refletindo assim, no seu desenvolvimento cognitivo (MATTOS e FARIA, 2011).

O trabalho do professor, com metodologias diversas nas atividades lúdicas, torna-se de grande importância para ajudar aos jovens na construção de conhecimentos, competências e desenvolvimento cognitivo. Kishimoto (2010) cita que,

O brincar é a atividade principal do dia a dia para as crianças. Nesta hora a criança toma decisões, mostra sentimentos, valores, conhecem a si, os outros e o mundo, repete ações prazerosas, partilha brincadeiras, mostra sua individualidade e identidade, explora o mundo dos objetos, das pessoas e da cultura, usa o corpo, os sentidos, os movimentos, as várias linguagens para experimentar situações que Ihe chamam a atenção para solucionar problemas e criar. Mas é no plano da imaginação que o brincar se destaca pela mobilização dos significados. Sua importância se relaciona com a cultura da infância que coloca a brincadeira como a ferramenta para se expressar, aprender e se desenvolver.

O jogo já é reconhecido por grande parte dos profissionais da educação, que o veem como um meio de estimular o raciocínio do jovem, estimulando sua aprendizagem. Tezani (2006) cita que,

O jogo não é simplesmente um "passatempo" para distrair os estudantes, ao contrário, corresponde a uma profunda exigência do organismo e ocupa lugar de extraordinária importância na educação escolar. Estimula o crescimento e o desenvolvimento, a coordenação muscular, as faculdades intelectuais, a iniciativa individual, favorecendo o advento e o progresso da palavra. Estimula o indivíduo a observar e conhecer as pessoas e as coisas do ambiente em que vive.

O jogo cria uma situação de regras que proporcionam uma zona de desenvolvimento proximal no estudante. Desse modo, este [...] comporta-se de forma mais avançada do que nas atividades da vida real e também aprende a separar objeto e significado [...] (OLIVEIRA, 1999, p. 67). A ação, durante o movimento do jogo, provoca espontaneidade. Isto causa estimulação suficiente para que o estudante transcenda a si mesmo (TEZANI, 2006). Durante o jogo, existe uma descontração e liberação de si para a atividade que esta praticando, a concentração aumenta e o estudante torna-se um jogador com intuito de vencer a partida.

Ele é libertado para penetrar no ambiente, explorar, aventurar-se e enfrentar, sem medo, todos os perigos. Dessa experiência integrada, surge o estudante ativo e participativo em um ambiente total, e aparecem o apoio e a confiança que Ihes permite desenvolver qualquer habilidade necessária para a comunicação dentro do 
jogo. Assim, os jogos proporcionam à criança adquirir o domínio da comunicação com os outros (TEZANI, 2006).

Os jogos favorecem o domínio das habilidades de com unicação, nas suas várias formas, facilitando a auto-expressão. Encorajam o desenvolvimento intelectual por meio do exercício da atenção, e também pelo uso progressivo de processos mentais mais complexos, como comparação e discriminação; e pelo estimulo à imaginação. Todas as vontades e desejos das crianças são possíveis de se rem realizados através do uso da imaginação, que a criança faz através do jogo (MATTOS e FARIA, 2011).

Fazendo um paralelo entre a situação da escola e os jogos, observo que os jogos aparecem em segundo plano quando falamos no processo de desenvolvimento, porém, a importância do jogo como recurso pedagógico, torna-se de grande valia, pois quando os estudantes estão jogando, existe uma articulação normal entre a prática executada e a teoria desenvolvida fazendo com que a aprendizagem se torne prazerosa.

Para Piaget o jogo é a construção do conhecimento, principalmente, nos períodos sensório motor e pré-operatório. Agindo sobre os objetos, os jovens, estruturam seu espaço, seu tempo, desenvolvem a noção de casualidade, chegando à representação e, finalmente à lógica (FARIA, 1994). Piaget (1971) classifica os jogos em "jogos de exercício, que se manifestam nos primeiros anos de vida, jogos simbólicos e jogos de regra", cita que [...] o jogo simbólico não é um esforço de submissão do sujeito ao real, mas, ao contrário, uma assimilação deformada da realidade do eu. De acordo com Macedo, Petty e Passos (2005, p.105):

Jogar não é simplesmente apropriar-se das regras. É Muito mais do que isso! A perspectiva do jogar que desenvolvemos relaciona-se com a apropriação da estrutura das possíveis implicações e tematizações. Logo não é somente jogar que importa (embora seja fundamental), mas refletir sobre as decorrências da ação de jogar, para fazer do jogo um recurso pedagógico que permite a aquisição de conceito e valores essenciais à aprendizagem.

É importante ressaltar que [...] os jogos educativos podem ser utilizados como Instrumentos de apoio contribuindo para a aprendizagem, sendo uma ferramenta de ensino transformando numa disputa divertida para o caminho do aprender [...] (BATISTA, e DIAS, 2012). Para Fialho (2008), [...] os jogos pedagógicos como ferramenta de ensino diz que [...] os jogos educativos, ou pedagógicos, com finalidades pedagógicas revelam a sua importância, pois promovem situações de ensino-aprendizagem e aumenta a construção do conhecimento. Considerando este ponto de entendimento, devemos ressaltar a maneira com que os jogos agem no desenvolvimento da agilidade, concentração e do raciocínio, contribuindo para o 
desenvolvimento intelectual. [...] Para isto necessita de ações como o pensar, tomar decisões, criar, inventar, aprender a arriscar e experimentar, estabelecendo um bom comportamento em grupo e também nas relações pessoais com o meio cultural na qual o sujeito está inserido (BATISTA, e DIAS, 2012).

Ao jogar, o estudante tem a oportunidade de resolver problemas, investigar e descobrir a melhor jogada, refletir e analisar as regras, estabelecendo relações entre os elementos do jogo e os conceitos matemáticos. Pode-se dizer que o jogo possibilita uma situação de prazer e aprendizagem significativa nas aulas de matemática (SMOLE, DINIZ e MILANI, 2007).

Smole, Diniz e Milani (2007), citam que o aprendizado com jogos pedagógicos [...] é um dos recursos que favorece o desenvolvimento da linguagem, diferentes processos de raciocínio e de interação, uma vez que durante um jogo, cada jogador tem a possibilidade de acompanhar o trabalho de todos os outros. Para as Orientações Curriculares Nacionais para o Ensino Médio (OCNEM) da área das Ciências da Natureza, Matemática e suas Tecnologias (BRASIL, 2006), os jogos pedagógicos são eficientes para a memorização e sugerem que há vários tipos de jogos que podem ser utilizados para instigar a memorização. Essas afirmativas fortalecem a discussão sobre uma metodologia que facilite a memorização de formulas relacionando-as a figuras geométricas, o que pode melhorar o aprendizado daqueles estudantes que possuem dificuldade no aprendizado de matemática. A metodologia de aprendizado com jogos pedagógicos se coloca como [...] o fio condutor no desenvolvimento das aulas de matemática, através dela, o estudante se apropria de conhecimentos obtidos pela observação e vivência dos fatos, adquirindo as competências e habilidades esperadas (SMOLE, DINIZ e MILANI, 2007).

A ação dos jogos pedagógicos no processo ensino-aprendizagem é visto a partir das avaliações, durante a aplicação do jogo como um instrumento avaliativo, [...] o estudante pode perceber se os conteúdos foram realmente assimilados, se sua aprendizagem obteve o êxito pretendido, se é necessário um estudo de determinado conteúdo e se ele está satisfeito com o que aprendeu durante sua aprendizagem (SILVA e AMARAL, 2011). Além do aprendizado proposto, podemos acrescentar a melhora na aceitação da disciplina em sala de aula, quando utilizamos de jogos pedagógicos para o ensino. É visto um divertimento com construção de conhecimentos e fortificação de amizade entre os estudantes.

O objetivo deste trabalho foi o de planejar e aplicar um jogo de dominó matemático para o ensino de geometria, englobando a temática de geometria plana e especial, como estratégia de complementação dos conteúdos programáticos de ensino de matemática do ensino médio. A partir da atividade pedagógica lúdica, busca-se promover reflexões a cerca das relações matemáticas estabelecidas no 
cálculo da área de figuras planas e de volume de sólidos de resolução, contribuindo para melhoria do processo de ensino-aprendizagem de geometria.

\section{Percurso Metodológico}

A pesquisa qualitativa sobre uma atividade pedagógica lúdica de ensino de geometria foi realizada no contexto do ensino técnico de nível médio. Os sujeitos desta pesquisa foram estudantes de uma turma do terceiro ano de ensino técnico integrado ao ensino médio de uma escola da Rede Federal de Educação Tecnológica do Espírito Santo, localizada na cidade de Santa Teresa, Estado do Espírito Santo, Brasil. Os dados foram produzidos a partir de observações, questionários e relatórios da aplicação do jogo de dominó matemático, além de alguns dados quantitativos.

Para realizada o estudo, foram organizados 4 grupos de estudantes, sendo 2 de 10 estudantes e 2 de 20 estudantes. Os quatro tratamentos iniciais foram compostos nas turmas, sendo escolhidos aleatoriamente 10 estudantes para jogar, e 20 estudantes para assessorar os jogadores, sendo que cada jogador teve 2 assessores. Estudantes que não participaram do jogo ou faltaram a aula no dia da atividade, formaram 0 quinto tratamento sendo considerado como testemunha. TA1 = jogadores, $\mathrm{TA} 2=$ assessores, $\mathrm{TB} 1=$ jogadores, $\mathrm{TB} 2=$ assessores, $\mathrm{TC}=$ testemunhas (ausentes). Foi considerado 10 repetições (estudantes), configurando um esquema fatorial simples 4 × 10 com testemunha adicional.

A primeira etapa da pesquisa constituiu em preparar as peças do jogo de dominó a partir da correlação entre figuras com área (tabela 1) e com volume (tabela 2). Essa etapa da pesquisa poderia ser realizada em conjunto com os estudantes, o que enriqueceria a atividade pedagógica oportunizando aos estudantes a serem protagonistas da construção do seu conhecimento matemático. Então, foram produzidas 101 peças de um dominó matemático contendo de um lado do dominó, uma figura geométrica, e do outro lado uma fórmula correspondente à área ou à volume. Na Figura 1 mostra alguns exemplos de peças de dominó matemático produzidos a partir da distribuição das figuras geométricas e as fórmulas.

A segunda etapa foi feita após o ensino tradicional em sala de aula. Foi aplicado um teste de múltipla escolha com 16 perguntas sobre o tema escolhido para todos os estudantes, ou seja, formulas das figuras planas e geométricas. Foi feito a avaliação das respostas e catalogadas como primeiro resultado da pesquisa, sem informar aos estudantes o resultado. 
Tabela 1: Relação das figuras geométricas e fórmulas utilizados na construção do jogo de dominó. Fonte: Dados de pesquisa.

\begin{tabular}{|c|c|c|c|}
\hline & Nome & Área \\
\hline 1 & Triângulo & Figura & S $=\frac{\mathrm{bxh}}{2}$ \\
\hline 2 & Quadrado & $\mathrm{S}=\mathrm{L}^{2}$ \\
\hline 3 & Losango & $\mathrm{S}=\frac{\mathrm{Dxd}}{2}$ \\
\hline 4 & Retângulo & & $\mathrm{S}=\mathrm{bxh}$ \\
\hline 5 & Trapézio & $\mathrm{S}=\frac{\mathrm{b} 1+\mathrm{b} 2) \mathrm{xh}}{2}$ \\
\hline 6 & Círculo & $\begin{array}{l}\mathrm{S}=\pi \cdot \mathrm{R}^{2} \\
\mathrm{C}=2 . \pi \cdot \mathrm{R}\end{array}$ \\
\hline
\end{tabular}

Observação: $S=$ área. $V=$ volume. $C=$ circunferência. $b=$ base. $h=$ altura. $L=$ lado. $R$ e $r=$ raio. $D$ e $d=$ diagonal.

Tabela 2: Relação das figuras geométricas e fórmulas utilizados na construção do jogo de dominó. Fonte: Dados de pesquisa.

\begin{tabular}{|c|c|c|c|}
\hline & Nome & Figura & Volume \\
\hline 1 & Cone & & $V=\frac{\pi \cdot R^{2} \cdot h}{3}$ \\
\hline 2 & Tronco de cone & & $V=\frac{\pi \cdot h\left(R^{2}+R r+r^{2}\right)}{3}$ \\
\hline 3 & Pirâmide & & $V=\frac{L 1 \cdot L 2 \cdot h}{3}$ \\
\hline 4 & Cubo & & $\mathrm{V}=\mathrm{L}^{3}$ \\
\hline 5 & Paralelepípedo & & $V=\mathrm{L} 1 . \mathrm{L} 2 . \mathrm{h}$ \\
\hline 6 & Esfera & & $V=\frac{4 \cdot \pi \cdot R^{3}}{3}$ \\
\hline 7 & Tronco de pirâmide & & $\mathrm{V}=\mathrm{h} \cdot \frac{(\mathrm{Sa}+\sqrt{\mathrm{Sa} \cdot \mathrm{Sb}}+\mathrm{Sb})}{3}$ \\
\hline 8 & Cilíndro & & $V=\pi \cdot R^{2} \cdot h$ \\
\hline
\end{tabular}

Observação: $\mathrm{S}$ = área. $\mathrm{V}$ = volume. $\mathrm{C}=$ circunferência. $\mathrm{b}=$ base. $\mathrm{h}$ = altura. $\mathrm{L}=$ lado. $\mathrm{R}$ e $\mathrm{r}=$ raio. $\mathrm{D}$ e $\mathrm{d}=$ diagonal. 
Figura 1: Alguns exemplos de peças do dominó matemático produzidas a partir da distribuição das figuras geométricas e das fórmulas.
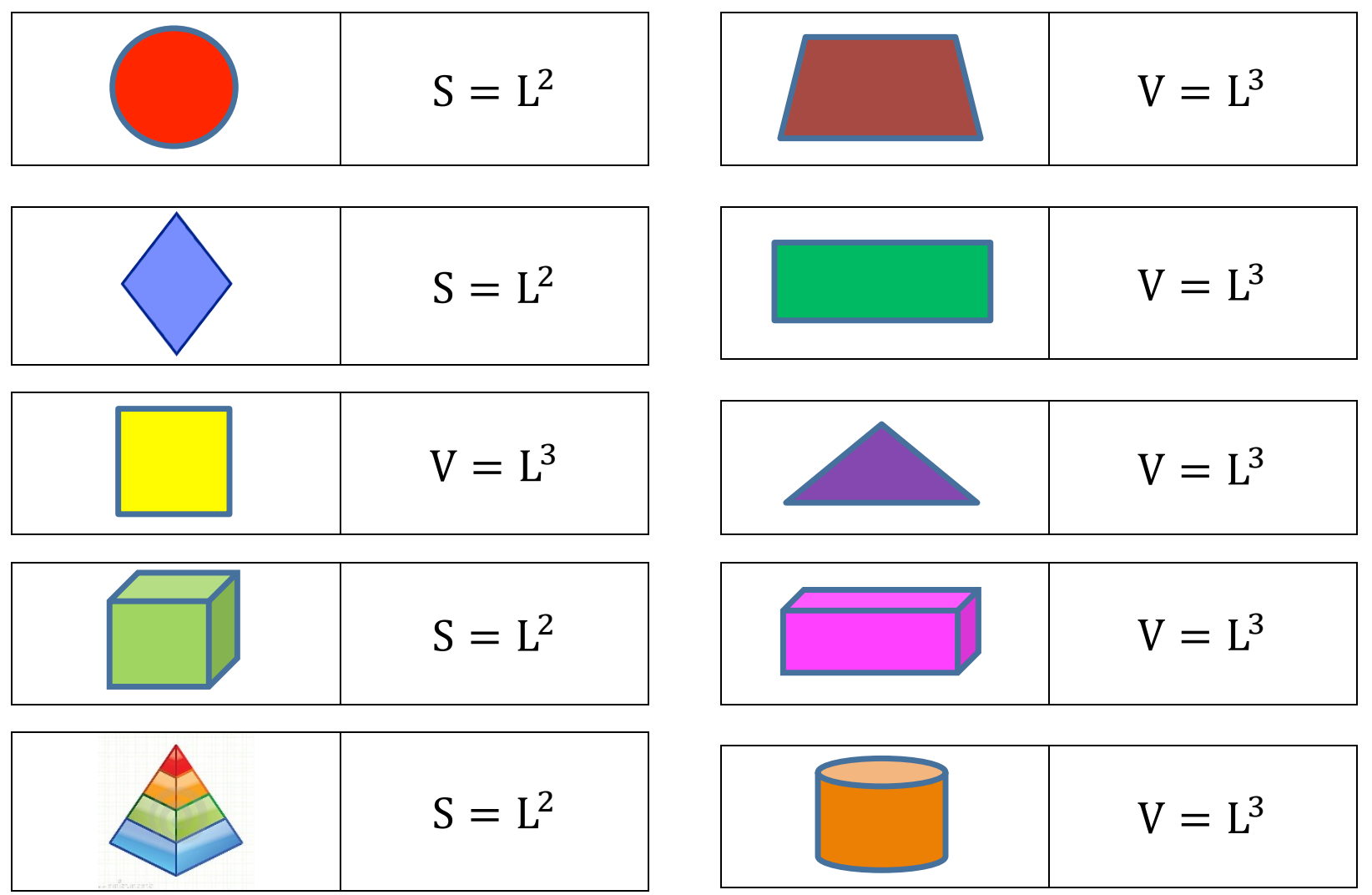

Sete dias após a primeira avaliação foi feito o jogo de dominó nas turmas, considerando que 10 estudantes de cada turma participaram do jogo e 20 não participaram, mas ficarão como assessores. Cada estudante recebeu 10 peças do dominó, e postos em círculo, os estudantes colocavam as peças seguindo a ordem em sentido horário, e na sequencia do jogo a peça que contemplasse a formula da figura exposta ou a figura representativa da fórmula apresentada na peça do dominó. O estudante que não tinha a peça representativa ou desconhecia a fórmula ou figura representativa, passava a vez para o próximo. O jogo terminava quando as peças de algum estudante se acabavam ou não existisse mais peça que contemplasse as opções de um ou outro lado da sequencia de peças utilizada.

Sete dias após a aplicação do jogo foi repetido à avaliação e catalogado os resultados para avaliação do aproveitamento, relacionando a primeira avaliação, a aplicação do jogo e a segunda avaliação, com vistas a avaliar a melhoria do aprendizado ou fixação das formulas para figuras geométricas planas e espaciais. Os dados foram coletados a partir das avaliações feitas antes e após o jogo e a avaliação dos dados se deu por comparação das notas tiradas antes e depois da aplicação d jogo a partir de análise estatística, sendo os dado submetidos à análise de variância e as médias comparadas pelo teste de Tukey em $5 \%$ de probabilidade. 


\section{Resultados e Discussão}

No desenrolar do jogo, o estudante que não tinha a peça representativa ou desconhecia a fórmula ou figura representativa, passava a vez para o próximo. Esta prática fazia com que o estudante que não tinha conhecimento da fórmula, passaria a conhecer a partir da colocação da peça pelo colega. A prática de passar a vez, tinha a função de fazer com que o estudante observasse qual a peça deveria ser colocada, se ele possuía ou não a peça correta, e se não colocou pelo desconhecimento da formula correta, ao ser colocado pelo colega o mesmo passaria a conhecer a formula correta para a figura posta, com inicio de aprendizado. A próxima vez que a figura foi exposta para o estudante, o mesmo já tinha conhecimento da fórmula e não mais passaria a vez.

Após a colocação da peça correta, o aprendizado ocorreu não só no estudante que passou a vez, mas também em todos os estudantes que desconheciam a fórmula daquela figura exposta, evitando que quando acontecesse com qualquer estudante, o conhecimento já tinha sido armazenado e não havia a necessidade de passar a vez. Considerando o desconhecimento de alguns estudantes ou mesmo a inexistência da peça correta para continuidade do jogo, o mesmo se posicionava com a finalização quando algum estudante não tinha mais peças, ou não havia mais peças que atendessem a resposta correta da figura ou formula contida nas últimas peças apresentadas no jogo.

Após aplicação da avaliação de conhecimentos dos estudantes das turmas A e B, considerando em um todo, e separando pelas categorias previamente selecionadas foi catalogado o resultado. Após a aplicação do jogo de dominó foi repetida a avaliação e catalogado os novos resultados considerando os estudantes participantes e não participantes das turmas $A$ e B, a média de todos os estudantes participantes e não participantes do jogo e a média total de todos os estudantes antes e depois do jogo em uma avaliação com 16 perguntas (tabela 3). Não podemos afirmar que muitos estudantes ou poucos estudantes tinham total conhecimento das fórmulas, considerando a média apresentada e o coeficiente de variação antes (23,68\%), cuja variação para maior ou menor estava em pouco mais de três respostas corretas ou incorretas.

Observa-se que não existe diferença significativa entre as médias dos estudantes participante do jogo e não participante do jogo, assim como a média geral, ou seja, antes do jogo todos os estudantes estavam em um patamar, com um coeficiente de variação de 23,68 \% e todos muito próximo da média geral. Após o jogo, houve um aprendizado geral de todos os estudantes participantes e não participantes, mostrando que o jogo atrai a atenção não somente dos jogadores, mas também dos estudantes que assistiam ao jogo havendo aprendizado de todos. 
Podemos observar que o coeficiente de variação caiu para 16,07 \%, mostrando que os estudantes se aproximaram da média geral, homogeneizando as notas e justificando a importância do jogo como metodologia de aprendizado de geometria.

Considerando que esta homogeneização de notas ocorreu com médias em um patamar superior as médias apresentadas na primeira avaliação, podemos afirmar que todos os estudantes melhoraram suas notas e que o reflexo do jogo no aprendizado foi positivo para toda a turma. Ainda neste quesito, podemos observar que não houve diferença significativa entre as notas, considerando as diferentes turmas e os estudantes que jogaram e não jogaram o dominó (tabela 3). A ausência de diferença significativa confirma a proposição do aprendizado de forma geral, propondo que a repetição do trabalho deverá atingir resultados semelhantes ao apresentado. O jogo de dominó apresenta-se como uma metodologia que atinge toda a turma, evita a desigualdade de aprendizado e estimula de forma homogênea toda a turma para aquisição de conhecimento do tema a ser apresentado.

Tabelas 3: Médias das avaliações feitas às diferentes turmas participantes e não participantes do jogo.

\begin{tabular}{ccc}
\hline Tratamentos & Antes & Depois \\
\hline TA participantes & $11,2666 \mathrm{a}$ & $13,4666 \mathrm{a}$ \\
TA não participantes & $10,8000 \mathrm{a}$ & $13,0000 \mathrm{a}$ \\
TB participantes & $11,3333 \mathrm{a}$ & $13,4000 \mathrm{a}$ \\
TB não participantes & $11,5333 \mathrm{a}$ & $13,4666 \mathrm{a}$ \\
TA + TB participantes & $11,3000 \mathrm{a}$ & $13,4333 \mathrm{a}$ \\
TA + TB não participantes & $11,1666 \mathrm{a}$ & $13,2333 \mathrm{a}$ \\
Part. + não Part. & $11,2667 \mathrm{a}$ & $13,4667 \mathrm{a}$ \\
\hline CV (\%) & 23,68 & 16,07 \\
\hline
\end{tabular}

Observação: Médias dos tratamentos seguidos da mesma letra em cada coluna, não diferem estatisticamente em $5 \%$ de probabilidade pelo teste de Tuckey. TA = Turma A, TB = Turma B. CV (\%) = Coeficiente de variação.

Quando comparamos as notas antes e depois do jogo, podemos observar a importância da metodologia aplicada. Em todos os quesitos houve diferença significativa, considerando os estudantes que jogaram, os que não jogaram e as médias. Observa-se na tabela 4, que em todos os casos ocorreu melhoria na fixação das fórmulas, com acréscimo de nota de forma significativa estatisticamente.

É de extrema importância à diminuição observada do coeficiente de variação, inicialmente, antes do jogo, tínhamos um coeficiente de variação de 23,68 \% (tabela 3), após o jogo a média final de todos os estudantes participantes e não participantes se aproximaram baixando o coeficiente de variação para 9,5 \% (tabela 4), mostrando que ocorreu fixação das fórmulas com um aprendizado, cuja média passou de 11,26 
acertos para 13,46 representando um acréscimo de 14,1\% médio de toda a turma, elevando e aproximando os conhecimentos sobre o tema, de todos os estudantes.

Os resultados apresentados mostram que todos os estudantes tiveram acréscimo na avaliação, com aproximação das menores notas com as maiores notas. Os estudantes mostraram que o jogo foi de grande valia na fixação do conteúdo proposto, quando após o jogo apresentaram resultados bem superiores na segunda avaliação, comparados aos resultados apresentados na primeira avaliação (tabela 4).

Tabela 4: Média da avaliação feita aos estudantes participantes antes e depois do jogo.

\begin{tabular}{cccccccc}
\hline Avaliação & TA part & $\begin{array}{c}\text { TA não } \\
\text { part. }\end{array}$ & TB part. & $\begin{array}{c}\text { TB não } \\
\text { part. }\end{array}$ & $\begin{array}{c}\text { TA + TB } \\
\text { par. }\end{array}$ & $\begin{array}{c}\text { TA + TB } \\
\text { não part. }\end{array}$ & $\begin{array}{c}\text { Part. + não } \\
\text { Part. }\end{array}$ \\
\hline Antes & $11,26 \mathrm{~b}$ & $10,80 \mathrm{~b}$ & $11,33 \mathrm{~b}$ & $11,33 \mathrm{~b}$ & $11,30 \mathrm{~b}$ & $11,16 \mathrm{~b}$ & $11,26 \mathrm{~b}$ \\
Depois & $13,46 \mathrm{a}$ & $13,00 \mathrm{a}$ & $13,40 \mathrm{a}$ & $13,46 \mathrm{a}$ & $13,43 \mathrm{a}$ & $13,23 \mathrm{a}$ & $13,46 \mathrm{a}$ \\
\hline CV $(\%)$ & 22,18 & 24,62 & 19,02 & 10,42 & 15,02 & 13,23 & 9,5 \\
\hline
\end{tabular}

Observação: Médias dos tratamentos seguidos da mesma letra em cada coluna, não diferem estatisticamente em $5 \%$ de probabilidade pelo teste de Tuckey. TA = Turma A, TB = Turma B, CV (\%) = Coeficiente de variação.

Corroboram com o resultado Smole, Diniz e Cândido (2007) quando citam que:

Ao jogar os estudantes têm a oportunidade de resolver problemas, investigar e descobrir a melhor jogada, refletir e analisar as regras, estabelecendo relações entre os elementos do jogo e os conceitos matemáticos. É evidente, portanto, que o jogo possibilita uma situação de prazer e aprendizagem significativa nas aulas de matemática.

Segundo Flemming (2004), [...] existem jogos que são verdadeiros exercícios de fixação. [...] Considera-se que o professor criativo, de acordo com seu ambiente, pode adaptar ou criar jogos como metodologia de ensino, para fixar qualquer tipo de conteúdo de Matemática. Aliados ao pensamento do autor, a metodologia apresentada estimula o aprendizado pois além de ser um jogo como outro qualquer, apresenta aos estudantes uma forma de aprender geometria, quando os mesmos consideram que além de brincar estão aprendendo. É característico do ensino de matemática as aulas expositivas, cujos conteúdos são repassados de forma teórica para que os estudantes apliquem em exercícios. Muitas vezes podemos observar a dificuldade de alguns estudantes em aplicar as fórmulas matemáticas ou mesmo memorizar estas fórmulas devido a grande quantidade existente de figuras geométricas. A prática do jogo tornou a aula mais agradável com maior atenção dos estudantes aos conteúdos propostos fazendo com que a aula expositiva apresentasse um caráter prático de expor conteúdos.

Rosa (2011, p.3) cita que [...] entendemos por aula expositiva aquela atividade cujo professor expõe um conteúdo, com a ajuda ou não de suportes tecnológicos: giz e quadro, transparências, epíscopo, slides, outras demonstrações, multimídia. O que 
caracterizamos como aula expositiva é a presença do professor expondo determinado conteúdo a um grupo de estudantes. A metodologia de aprendizado com jogo de dominó mescla o que entendemos por aula expositiva com aula prática, a participação do estudante no jogo, e a prática de jogar, colocam a metodologia como centro de ensino-aprendizagem, trazendo ao estudante o conteúdo que se quer ensinar.

\section{Conclusão}

Este trabalho se concentrou no planejamento e aplicação de um jogo de dominó matemático visando à temática de geometria plana e especial, como estratégia complementar da abordagem de conteúdos programáticos de matemática do ensino médio. Buscou-se promover reflexões a cerca das relações matemáticas estabelecidas no cálculo da área de figuras planas e de volume de sólidos de resolução a partir de uma atividade lúdica, contribuindo para melhoria do processo de ensino-aprendizagem de geometria.

Embora algumas etapas de produção do jogo tenham sido feitas somente com a participação dos investigadores, essas poderiam ser realizada em conjunto com os estudantes, o que enriqueceria a atividade pedagógica oportunizando aos estudantes a serem protagonistas da construção do seu conhecimento matemático. Vale citar que o estudo sobre a aplicação do jogo matemático não abordou a contextualização dos conteúdos programáticos, embora isso tenha acontecido nos debates entre estudantes durante o processo de aplicação do jogo de dominó.

A metodologia de ensino de geometria a partir do jogo de dominó apresentouse como uma forma lúdica, de fácil aplicação e positiva para o aprendizado dos conteúdos de matemática. Os resultados indicaram que a metodologia contribuiu no aprendizado dos estudantes que praticaram o jogo de dominó, o que foi constatado nos resultados obtidos nos coeficientes de variação da avaliação feita antes e depois do jogo.

A valorização da metodologia de ensino de matemática se deu a partir do aprendizado lúdico, agradável e com de boa aceitação por parte dos estudantes. Vale ressaltar que a metodologia do jogo de dominó discutida neste trabalho foi utilizada no aprendizado complementar de geometria euclidiana. Porém, esta metodologia pode ser aplicada em outras áreas do conhecimento da educação básica, além da matemática, como as ciências naturais (física, química e biologia) e situações interdisciplinares com a geografia e a história, envolvendo perpassando por discussões da matemática. 


\section{Referências}

BATISTA, D. A. DIAS, C. L. O processo de ensino e de aprendizagem através dos jogos educativos no ensino fundamental. Colloquium Humanarum. vol. 9, n. Especial, jul-dez, 2012.

BRASIL. Diretrizes Curriculares Nacionais. Brasília - DF: Ministério da Educação, 2013.

BRASIL. Orientações curriculares para o ensino médio. Volume 2. Ciências da natureza, matemática e suas tecnologias. Secretaria de Educação Básica. Brasília-DF: Ministério da Educação, Secretaria de Educação Básica, 2006. 135 p.

FARIA, A. R. O pensamento e a linguagem da criança segundo Piaget. 2 ed. São Paulo: Ática, 1994.

FLEMMING, D. M. Criatividade e Jogos Didátios. Recife: ENEM. 2004.

KISHIMOTO, T. M. Jogo, brinquedo, brincadeira e a educação. 13ed. São Paulo: Cortez, 2010. 207p.

MACEDO, I.; PETTY, A. L. S.; PASSOS, N. C. Os jogos e o lúdico na aprendizagem escolar. Porto Alegre: Artmed, 2005.

MATTOS, R. C. F.; FARIA, M. A. Jogo e Aprendizagem. Revista Eletrônica Saberes da Educação, V. 2., 2011.

OLIVEIRA, M. K. Vygotsky: aprendizado e desenvolvimento, um processo sóciohistórico. 4. ed. São Paulo: Scipione, 1999.

PIAGET, J. A formação do símbolo na criança, imitação, jogo, sonho, imagem e representação de jogo. São Paulo: Zahar, 1971.

ROSA, P. R. S. A Aula Expositiva: Instrumentação para o ensino de Ciências. p. 110. UFMS, 2011.

SILVA, T. C.; AMARAL, C. L. C. Jogos e avaliação no processo ensino-aprendizagem: uma relação possível. Revista de ensino ciência e matemática, v. 2, n. 1, p.1-8, jan/jun 2011.

SMOLE, K. S.; DINIZ, M. I.; CÂNDIDO, P. T. Jogos de matemática de 1a 50 ano. In série. Cadernos do Mathema Ensino Fundamental. Porto Alegre: Artmed, 2007. $150 \mathrm{p}$.

SMOLE, K.S.; DINIZ, M.I.; MILANI, E. Jogos de matemática do $6^{\circ}$ ao $9^{\circ}$ ano. Cadernos do Mathema. Porto Alegre: Artmed 2007. 
TEZANI, T. C. R. O jogo e os processos de aprendizagem e desenvolvimento: aspectos cognitivos e afetivos. Educação em Revista, v.7, n.1/2, p. 1-16, Marília, 2006. 